\title{
Impact of old age on resectable colorectal cancer outcomes
}

\author{
Jianfei Fu ${ }^{1}$, Hang Ruan ${ }^{2}$, Hongjuan Zheng ${ }^{1}{ }^{\text {, Cheng Cai }}{ }^{2}$, Shishi Zhou ${ }^{1}$, Qinghua Wang ${ }^{1}$, Wenbin Chen ${ }^{3}$, \\ Wei Fu ${ }^{4}$, Jinlin Du Corresp. 2 \\ ${ }^{1}$ Department of Medical Oncology, Jinhua Hospital, Zhejiang University School of Medicine, Jinhua, Zhejiang, P.R.China \\ 3 Department of Colorectal Surgery, The First Affiliated Hospital, Zhejiang University School of Medicine, Hangzhou, Zhejiang, P.R.China \\ 4 Division of Oncology, Johns Hopkins University School of Medicine, Baltimore, Maryland, United States of America \\ Corresponding Author: Jinlin Du \\ Email address: dj19090@163.com
}

Objective: This study was performed to identify a reasonable cutoff age for defining older patients with colorectal cancer (CRC) and to examine whether old age was related with increased colorectal cancerspecific death (CSD) and poor colorectal cancer-specific survival (CSS).

Methods: 76,858 eligible patients from the Surveillance, Epidemiology and End Results (SEER) database were included in this study. The Cox proportional hazards regression model and the Chow test were used to determine a suitable cutoff age for defining the older group. Furthermore, a propensity score matching analysis was performed to adjust for heterogeneity between groups. A competing risk regression model was used to explore the impact of age on CSD and non-colorectal cancer-specific death (non-CSD). Kaplan-Meier survival curves were plotted to compare CSS between groups. And a Cox regression model was used to validate the results. External validation was performed on data from 1998 to 2003 retrieved from the SEER database.

Results: Based on a cutoff age of 70 years, the examined cohort of patients was classified into a younger group ( $n=51,915,<70$ years of old) and an older group ( $n=24,943, \geq 70$ years of old). Compared with younger patients, older patients were more likely to have fewer lymph nodes sampled and were less likely to receive chemotherapy and radiotherapy. When adjusted for other covariates, agedependent differences of 5-year CSD and 5-year non-CSD were significant in the younger and older groups (15.84\% and $22.42 \%, \mathrm{P}<0.001 ; 5.21 \%$ and $14.21 \%, \mathrm{P}<0.001)$. And an age of $\geq 70$ years remained associated with worse CSS comparing with younger group (subdistribution hazard ratio [SHR], 1.51 [95\% confidence interval [Cl], 1.45-1.57], $\mathrm{P}<0.001$ ). The Cox regression model as a sensitivity analysis had a similar result. External validation also supported an age of 70 years as a suitable cutoff, and this older group was associated with having reduced CSS and increased CSD.

Conclusions: Seventy is a suitable cutoff age to define those considered as having elderly CRC. Elderly CRC was associated with not only increased non-CSD but also with increased CSD. Further research is needed to provide evidence of whether cases of elderly CRC should receive stronger treatment if possible. 
1 Impact of old age on resectable colorectal cancer outcomes

\section{Jianfei Fu ${ }^{1,}{ }^{*}$, Hang Ruan ${ }^{2,}$, Hongjuan Zheng ${ }^{1}$, Cheng Cai2, Shishi Zhou ${ }^{1}$, Qinghua}

3 Wang', Wenbin Chen ${ }^{3}$, Wei Fu ${ }^{4}$, Jinlin $\mathrm{Du}^{2}$

$4{ }^{*}$ These authors contributed equally to this work

$5{ }^{1}$ Department of Medical Oncology, Jinhua Hospital, Zhejiang University School of Medicine,

6 Jinhua, Zhejiang 321000, P.R.China

72 Department of Colorectal Surgery, Jinhua Hospital, Zhejiang University School of Medicine,

8 Jinhua, Zhejiang 321000, P.R.China

$9{ }^{3}$ Department of Colorectal Surgery, The First Affiliated Hospital, Zhejiang University School of

10 Medicine, Hangzhou, Zhejiang 310003, P.R.China

$11{ }^{4}$ Division of Oncology, Johns Hopkins University School of Medicine, Baltimore, Maryland,

12 United States of America

14 Corresponding author:

15 Jinlin $\mathrm{Du}^{2}$

16 Department of Colorectal Surgery, Jinhua Hospital, Zhejiang University School of Medicine,

17 Jinhua, Zhejiang 321000, P.R.China

18 E-mail address: dj19090@163.com 
ABSTRACT

Objective: This study was performed to identify a reasonable cutoff age for defining older patients with colorectal cancer (CRC) and to examine whether old age was related with increased colorectal cancer-specific death (CSD) and poor colorectal cancer-specific survival (CSS).

Methods: 76,858 eligible patients from the Surveillance, Epidemiology and End Results (SEER) database were included in this study. The Cox proportional hazards regression model and the Chow test were used to determine a suitable cutoff age for defining the older group. Furthermore, a propensity score matching analysis was performed to adjust for heterogeneity between groups. A competing risk regression model was used to explore the impact of age on CSD and noncolorectal cancer-specific death (non-CSD). Kaplan-Meier survival curves were plotted to compare CSS between groups. And a Cox regression model was used to validate the results. External validation was performed on data from 1998 to 2003 retrieved from the SEER database.

Results: Based on a cutoff age of 70 years, the examined cohort of patients was classified into a younger group $(n=51,915,<70$ years of old) and an older group ( $n=24,943, \geq 70$ years of old). Compared with younger patients, older patients were more likely to have fewer lymph nodes sampled and were less likely to receive chemotherapy and radiotherapy. When adjusted for other covariates, age-dependent differences of 5-year CSD and 5-year non-CSD were significant in the younger and older groups $(15.84 \%$ and $22.42 \%, \mathrm{P}<0.001 ; 5.21 \%$ and $14.21 \%, \mathrm{P}<0.001)$. And an age of $\geq 70$ years remained associated with worse CSS comparing with younger group (subdistribution hazard ratio [SHR], 1.51 [95\% confidence interval [CI], 1.45-1.57], P $<0.001$ ). The Cox regression model as a sensitivity analysis had a similar result. External validation also 
40 supported an age of 70 years as a suitable cutoff, and this older group was associated with having

41 reduced CSS and increased CSD.

42 Conclusions: Seventy is a suitable cutoff age to define those considered as having elderly CRC.

43 Elderly CRC was associated with not only increased non-CSD but also with increased CSD.

44 Further research is needed to provide evidence of whether cases of elderly CRC should receive

45 stronger treatment if possible.

46 Keywords: Colorectal cancer; old; age; cancer specific death; SEER; competing risk regression

\section{INTRODUCTION}

49 Age-specific risk rises markedly in old age, and as mortality from heart disease and other non-

50 cancer causes decrease, this leaves the elderly population at high risk for developing bowel

51 cancer(Papamichael et al. 2009). The increasing incidence of colorectal cancer (CRC) in the

52 segment of the population $>70$ years of age necessitates an examination of what type of

53 treatment is most appropriate for these patients with colorectal cancer. Approximately $60 \%$ of

54 CRC patients are $>70$ years of age at the time of diagnosis, and $43 \%$ are $>75$ years of

55 age(Papamichael et al. 2009). It remains controversial whether so-called "elderly CRC" exhibits

56 a differential prognosis compared to younger CRC and whether age (i.e., old) is an independent

57 prognostic factor. There is now a general consensus that those in the old population possess a

58 high frequency of frailty and comorbidities, exhibiting increased mortality from other causes

59 among those with CRC. However, it remains unknown whether old patients have an increased

60 incidence of colorectal cancer specific death (CSD). A previous report demonstrated that older 
61 patients with CRC who survived the first year after surgery exhibited the same overall cancer-

62 related survival as did younger patients(Dekker et al. 2011). Of note, chronological age is

63 distinct from biological age(Odden et al. 2012; Robinson et al. 2009). The definition of 'elderly'

64 differs, being given as anything between $>65$ years and $>80$ years in different studies(Seymour

65 2004; Twelves et al. 2005). As such, it is difficult to know whether 70 years is a reasonable

66 cutoff age to safely extrapolate these results or whether the decision should depend on the

67 physical and functional status of the patient rather than just on chronological age.

68 Unlike younger patients with CRC, wherein more reliable evidence-based guideline based on

69 clinical trials are available, older patients are still in need of increased evidence to guide clinical

70 practices due to most trials excluding older patients with CRC. We used big data to explore the

71 impact of old age on CRC prognoses to help guide clinical practice in the treatment of elderly

72 CRC patients.

MATERIALS \& METHODS

\section{Data}

Data on colon cancer records from 18 cancer registries in the National Cancer Institute's

Surveillance, Epidemiology and End Results (SEER) Cancer database based on the November

2015 submission were collected. SEER.Stat software was utilized to identify patients with resectable stage I-III CRC, and information regarding chemotherapy was obtained by submitting a special data request to the SEER program.

Cohort inclusion criteria were as follows: (1) Years of diagnosis from 2004 to 2011. (2) Patients 
82 diagnosed with stage I-III CRC. (3) Patients received surgery (Surgery code 30-80). (4)

83 Histological type ICD-O-3 was limited to $8140 / 3,8480 / 3,8481 / 3$ and 8490/3. Exclusion criteria

84 were as follows: (1) Patients lacking documentation of age at diagnosis, gender, race, marital

85 status, differentiated grade, and classification T. (2) Patients younger than 20 years or older than

80 years. (3) Patients with multiple primary tumors. (4) Patients who survived less than one month from diagnosis. (5) Cause of death was unknown. (6) Number of lymph nodes sampled was unknown. (7) The number of positive lymph nodes was unknown.

\section{Variables declaration}

Patients were classified into younger ( $<70$ years old) and older ( $\geq 70$ years old) groups based on the defined cutoff age of 70 years. Race was divided into white, black and other. Marital status was categorized as married, single (never married, unmarried or domestic partner) or divorced (separated, widowed and divorced). Tumor location was grouped into left CRC or right CRC. Left colorectal cancer includes the rectum, rectosigmoid junction, sigmoid colon, descending colon and splenic flexure. Right colorectal cancer includes the transverse colon, hepatic flexure, ascending colon, cecum, and appendix. Histological type was categorized as adenocarcinoma, mucinous adenocarcinoma, or ring signet cell cancer. All cases were regrouped according to the 7th American Joint Committee on Cancer (AJCC) TNM staging system. Number of lymph nodes (nLN) sampled was regrouped as $0,1-3,4-6,7-11$, and $\geq 12$. The variable chemotherapy was classified as chemotherapy "yes" or "no/unknown" according to the SEER program(Noone et al. 2016). 


\section{Statistical analysis}

105 The restrict cubic spline function, "RCS", with three knots was used to transform the continuous variable of age. The "rcspline.plot" function provided plots of the estimated restricted cubic

107 spline function relating a single predictor (age) to the response for a Cox model. The Chow test method (Fstats and breakpoints in strucchange package) was used to explore a suitable cutoff value for age to define elderly CRC. Differential distribution of clinicopathological characteristics between younger and older subgroups was indicated by standardized difference (SD)(Austin 2009). Propensity scores were used to balance the difference of distribution between younger and older groups on sociodemographic and clinical characteristics. Matchit package in

$\mathrm{R}$ software was used as the nearest method with ratio 1:1. Cancer-specific death (CSD) and noncancer-specific death (non-CSD) were considered the primary endpoint and were calculated by the Gray test(Howlader et al. 2014). The secondary endpoint was CSS. The non-CSD referred to the dead due to other causes. When CSDs were calculated, follow up time was calculated from

117 the date of diagnosis to the date of death from colorectal cancer. Alive were defined as censored, and the non-CSD was considered a competing event. The subdistribution hazard ratio (SHR) of variables for cause-specific death was estimated using the Fine and Gray proportional hazard model(Fine \& Gray 1999). As a comparison, the hazard ratio (HR) of variables was also estimated with a Cox proportional hazards model. 
124

125

126

127

128

129

130

131

132

133

134

135

136

137

138

139

140

141

142

143

mean or prevalence of a covariate between groups(Normand et al. 2001). When a two-sided $P$ value was less than 0.05 , the difference was considered statistically significant. All statistical analyses were performed using R software (version 3.3.2).

\section{RESULTS}

\section{Baseline characteristics and identification of the old age cutoff value}

76,858 eligible patients were included in this cohort. The endpoint date for follow up was November 2013, and the median follow-up time was 55.0 months (range 1.0-119.0 months).

The median age of patients was 64 years (IQR 20-80 years). With age as a continuous variable, the hazard ratio (HR) of CSS was 1.54 (95\% CI 1.39- 1.69, $P<0.001)$. The HR of CSS slowly increased before 70 years of age, and then increased significantly after 70 years according to the Cox model (Fig. 1).

\section{Clinicopathological features of old colorectal cancer patients}

Based on the cutoff age of 70 years, the test cohort of patients was classified into the following two groups: younger group ( $\mathrm{n}=51,915,<70$ years of old) or older group $(\mathrm{n}=24,943, \geq 70$ years of old). Older patients exhibited a high frequency of male, Caucasians, right CRC, mucinous carcinoma, more poorly differentiated grade and earlier stage. Detailed clinicopathological characteristics of the chemotherapy subgroups are presented in Table 1. Compared with younger patients, older patients were more likely to have fewer lymph nodes sampled and were less likely 
144 to receive chemotherapy and radiotherapy.

145 The sample size of patients in the older group was obviously fewer than in the younger 146 group, and these groups had different clinical characteristics, so a method for propensity score 147 matching (PSM) was used to balance differences in baseline characteristics and generate a 148 corrected test cohort. Most covariates were well balanced between younger and older groups in 149 the corrected test cohort (Table 1).

150

151

3. Competing risk regression model was used to explore the impact of age on CSD and non-

152

153

154

155

156

157

\section{CSD}

14,425 (18.77\%) and 6,982 (9.08\%) patients died of CSD and non-CSD, respectively. The corrected test cohort after PSM, a total of 47,616 patients analyzed, showed that 9,273 (19.47\%) and 5,514 (11.58\%) patients died of CSD and non-CSD, respectively. The 5-year CSD in the younger and older groups were $15.84 \%$ and $22.42 \%$, respectively, and were significantly different $(P<0.001)$. The 5-year non-CSD in the younger and older groups were 5.21\% and 14.21\%, respectively, and were also significantly different $(P<0.001)$ (Fig. 2). Univariate and multivariate analyses demonstrated that old age was associated with CSD (Table 2)).

\section{Subgroup analysis by characteristics in raw data}

Subgroup analyses were performed based on gender, race, differentiated type, pathological type, $\mathrm{T}$ and $\mathrm{N}$ classification, $\mathrm{nLN}$, TNM stage, chemotherapy and radiotherapy in the raw data. In all subsets (except for ring signet cell cancer), patients in the older group exhibited poorer prognosis 
165

166

167

168

169

170

171

172

173

174

175

176

177

178

179

180

181

182

183

184

185

compared with those in the younger group. (Fig. 3). Furthermore, we performed an interaction analysis using Cox model between the therapy and age in stage III patients in raw data (Fig. S2).

The results showed that the older group benefited more from chemotherapy than younger group $(P$ value $=0.001)$. However, the interaction between radiotherapy and old was not significant $(P$ value $=0.328)$

\section{Univariate and Multivariate Cox regression analysis}

A Kaplan-Meier survival curve for CSS is presented in Fig. 4. Univariate analysis showed a HR for CSS of older patients of 1.57 (95\% CI 1.51-1.64, $P<0.001$, reference to younger group) in the corrected cohort. Multivariate analysis showed that the HR for CSS in older patients was 1.64 (95\% CI 1.57-1.70, $P<0.001$, reference to younger group) (for detailed data see Table 3) (raw data are shown in Table S1).

\section{External validation}

In the validated cohort, 66,946 patients from 1998 to 2003 were retrieved from the SEER dataset. The corrected validated cohort after PSM was used to validate the above results. The relationship between age and colorectal cancer-specific death for Cox model presented a single arm " $U$ " shape (Fig. S1). Seventy was still the cutoff age in the validated cohort. Detailed clinicopathological characteristics are presented in Table S2. In the corrected validated cohort, there were few differences in the distribution of different clinicopathological factors between older and younger groups (see Table S2). Univariate and multivariate analysis showed that old 
186 age is related with poor CSS (based on the Cox model) and increased CSD (based on the 187 competing risk model) (see Table S3).

\section{DISCUSSION}

The statistical methods "RCS" and "Chow test" were used to determine that 70 years is a reasonable cutoff age to define elderly CRC. Elderly CRC included a high frequency of male patients, right site $\mathrm{CRC}$, mucinous carcinoma, more poorly differentiated grades and earlier stages. There were fewer than 12 lymph nodes sampled and earlier stages in the older group.

After eliminating the distribution difference between older and younger groups by PSM, elderly

CRC had worse outcomes (CSS), and age was shown to be an independent prognostic factor, while elderly CRC was related with increased non-CSD as well as CSD. In almost all subgroups, elderly CRC exhibited worse outcomes. The external cohort validated the reasonability of 70 years as a cutoff age to define elderly $\mathrm{CRC}$ and further confirmed that elderly CRC exhibited worse outcomes (CSS or CSD).

In clinical practice, the optimal cutoff age is anticipated to define elderly CRC. The screening program for CRC defined 65 years as a cutoff age(Papamichael et al. 2009). In other previously published studies(McCleary et al. 2013a; Merchant et al. 2017; Tournigand et al. 2012; Twelves et al. 2005), 70 years was adopted as a cutoff age. Updated SEER-Medicare analysis data and three population-based data sets conducted by Sanoff et al(Sanoff et al. 2012) showed that only $44 \%$ of the 5941 patients evaluated received adjuvant chemotherapy within 3 months of surgical resection for stage III CRC. In their study, 65 years was used to define elderly 
207 CRC. In clinical trials, 75 years was more frequently set as the upper limit; therefore, a more

208 real-world data analysis adopted 75 years as their cutoff age(van Erning et al. 2013). We

209 concluded that 70 years should be adopted as a suitable cutoff age.

210 Once the cutoff age was defined, we found that elderly CRC exhibited a significantly

211 different outcome than young CRC. Several studies have shown that elderly CRC has

212 comparable outcomes compared with younger CRC. Dekker et al.'s(Dekker et al. 2011) study

213 showed that if one excluded death due to operation comorbidities (mostly death occurring one

214 year after an operation), there were rather similar outcomes between the younger and older

215 groups. Late period survival was similar between older and younger subgroups for resectable

216 CRC(Dekker et al. 2011). One Canadian study(Merchant et al. 2017) showed that elderly CRC

217 did exhibit worse prognosis and was associated with a high Charlson index. In their study, they

218 did not differentiate between CSD and non-CSD; therefore, their study did not conclude that

219 elderly CRC was related with increased CSD. In our study, we used a competing risk model to

220 distinguish non-CSD and CSD. Furthermore, we report that elderly CRC is associated with

increased CSD. In late period follow up, the Gray's cumulative events curve on CSD separated

more clearly, indicating that elderly $\mathrm{CRC}$ is associated with worse $\mathrm{CSD}$, which might be due to

different register periods. Therefore, we included patients from different periods as a validation

cohort. The validation cohort also confirmed that elderly CRC was not only related with

increased CSD but also with increased non-CSD. Our study confirmed that elderly CRC exhibits

poorer prognosis from several aspects. Except for external validation and competing risk mode, 
228

229

230

231

232

233

234

235

236

237

238

239

240

241

242

243

244

245

246

247

248

differential distribution.

Worse outcome of elderly $\mathrm{CRC}$ is multi-faceted. Fewer numbers of lymph nodes were removed from those with elderly $\mathrm{CRC}$, contributing to residual tumor matter in the anticipated dissection region (mean to R1 resection) and underestimated tumor stages. Moreover, elderly CRC exhibited less capacity to endure stronger treatments, resulting in lower intensity adjuvant chemotherapy and radiotherapy. Our correlation analysis indicated that elderly CRC was related with lower incidence of chemotherapy. The outcome of less frequent chemotherapy was similar to findings in previous studies(Abraham et al. 2013; Kahn et al. 2010; van Erning et al. 2014; van Erning et al. 2013). Finally, a CRC screening program resulted in the increased detection of precancerous lesions (such as polyps) that were treated in the old population. If the CRC diagnosis escaped screening, there was typically increased short-term carcinogenesis and more aggressive tumor behavior. Another study showed that elderly CRC had a greater index of genetic mutations and that the incidence of BRAF mutations was higher. Berg et al. indicated that CIMP tumors are more common in the older population, who also have a higher rate of KRAS and BRAF mutations(Berg et al. 2010).

Elderly CRC with worse outcomes might require stronger treatments; however, a previous study suggested that elderly $\mathrm{CRC}$ does not require enhanced treatment. Many elderly patients will benefit from radical treatment approaches, but others will not, and in some cases, nonoperative "palliative" management should be offered, even though the cancer is "curable".

47 Guidelines from the International Society of Geriatric Oncology (SIOG) did not recommend that elderly CRC patients regularly receive adjuvant chemotherapy with limited evidence to support 
249 the benefit from such strategy(Papamichael et al. 2009). MOSAIC: lyses showed there to be no 250 statistically significant benefit conferred by addition of oxaliplatin in terms of disease-free 251 survival (DFS) or OS for older patients (70-75years), although female patients 70-75 years of 252 age exhibited the same oxaliplatin benefit as did younger patients(Tournigand et al. 2012). 253 Interestingly, the DFS and OS benefits in patients 70-75 years were similar to those of younger 254 patients for the first 3 years of follow-up but were lost later on due to deaths from other causes(Andre et al. 2009; Tournigand et al. 2012). NSABP-C-07: Patients $\geq 70$ years failed to derive a statistically significant DFS or OS benefit from addition of oxaliplatin(Tournigand et al. 2012). Indeed, those patients receiving FLOX had poorer survival, which was attributed to toxicity. XELOXA (NO16968): the benefits observed for XELOX were maintained, although to 259 a lesser degree in patients $\geq 65$ and $\geq 70$ years of age, in contrast to the results from MOSAIC and NSABP-C-07 trials(Twelves et al. 2012). Meta-analysis of ACCENT did not support that patients $\geq 70$ years receive additional oxaliplatin chemotherapy(McCleary et al. 2013a; McCleary et al. 2013b). In contrast, from an analysis using real-world data, almost all showed that elderly CRC can benefit from adjuvant chemotherapy with acceptable toxicity(Abraham et al. 2013; Kahn et al. 2010; Sanoff et al. 2012; Sanoff \& Goldberg 2007; Schrag et al. 2001; van Erning et al. 2014; van Erning et al. 2013). One study using SEER-Medicare data indicated that elderly CRC tolerated chemotherapy well, exhibiting worse prognosis due to reduced treatment(Sanoff et al. 2012). More recent studies showed that with the technological development of laparoscopic surgery and enhanced recovery programs after surgery (ERAS), elderly CRC could tolerate operation well. The literature suggests that elderly patients benefit from multimodal 
270

271

272

273

274

275

276

277

278

279

280

281

282

283

284

285

286

287

288

289

290

rehabilitation programs or ERAS in the same way as younger patients(van Steenbergen et al. 2013). Our interaction analysis between the therapy and age in stage III patients showed that the older group benefited more from chemotherapy than younger group $(P$ value $=0.001)$. And compared with younger patients, older patients were more likely to have fewer lymph nodes sampled and were less likely to receive chemotherapy and radiotherapy. Therefore, current treatment paradigms in the older group may be insufficient. As life expectancy increases, more effective treatments are necessary for the old population.

To reduce bias as much as possible, we used PSM to balance clinicopathological characteristics and used a competing risk model to exclude impact from non-CSD. Finally, we confirmed that elderly CRC was related with more CSD and non-CSD. Age(old) is an independent factor to predict increased CSD. And the Cox model as a sensitivity analysis also had a similar result. Our analysis provides more evidence for elderly CRC receiving stronger adjuvant chemotherapy. The above conclusions can only be acquired from real world data analysis rather than from clinical trials alone.

As a retrospective study, it is impossible to avoid all bias for patient selection. There are several limitations inherent to the database used in the current study. The Charlson index is not available in SEER data. Though the SEER-Medicare can retrieve Charlson index, only patients older than 60 years are registered in their dataset. The Charlson index is strongly related with non-CSD in the old population. Competing risk models can effectively eliminate the impact from unavailable Charlson index. BMI is also an important influence factor in the colorectal cancer specific mortality(Kroenke et al. 2016). However, the information about BMI in the SEER data 
291 is not available. Furthermore, insurance status is an independent risk factor both for advanced

292 disease in their cancer diagnosis and for cancer mortality(Rosenberg et al. 2015), and uninsured

293 patients more often have higher T, N and M stage(Amini et al. 2016). But the insurance recode

294 variable is only available from 2007 in the SEER database. Additionally, comorbidities and

295 detailed information about driver gene mutations (KRAS or BRAF) is not available. The variable

296 chemotherapy is only classified as chemotherapy "yes" or "no/unknown" since SEER treatment

297 information cannot accurately distinguish between "no treatment" and "unknown"(Noone et al.

298 2016). Furthermore, the sensitivity of SEER chemotherapy data is only $72.1 \%$ (Noone et al.

299 2016), as the detailed regime and duration of chemotherapy is not available in the SEER dataset.

300

301

\section{CONCLUSIONS}

302

303

304

305

306

307

308

309

310

311

312

313

314

In summary, 70 years is a suitable cutoff age to define elderly CRC. Elderly CRC is associated with not only increased non-CSD but also increased CSD. This SEER-based analysis provides further evidence that current chemotherapy in the elderly may be insufficient. Additional research is required to investigate whether elderly CRC will receive stronger treatment if possible.

\section{Abraham A, Habermann EB, Rothenberger DA, Kwaan M, Weinberg AD, Parsons HM,} Gupta P, and Al-Refaie WB. 2013. Adjuvant chemotherapy for stage III colon cancer in the oldest old: results beyond clinical guidelines. Cancer 119:395-403. DOI 10.1002/cncr.27755

Amini A, Jones BL, Yeh N, Guntupalli SR, Kavanagh BD, Karam SD, and Fisher CM. 
2016. Disparities in disease presentation in the four screenable cancers according to health insurance status. Public Health 138:50-56. DOI 10.1016/j.puhe.2016.03.014

Andre T, Boni C, Navarro M, Tabernero J, Hickish T, Topham C, Bonetti A, Clingan P, Bridgewater J, Rivera F, and de Gramont A. 2009. Improved overall survival with oxaliplatin, fluorouracil, and leucovorin as adjuvant treatment in stage II or III colon cancer in the MOSAIC trial. J Clin Oncol 27:3109-3116. DOI 10.1200/jco.2008.20.6771

Austin P. 2009. Using the Standardized Difference to Compare the Prevalence of a Binary Variable Between Two Groups in Observational Research. Communications in Statistics - Simulation and Computation 38:1228-1234. DOI

Berg M, Danielsen SA, Ahlquist T, Merok MA, Agesen TH, Vatn MH, Mala T, Sjo OH, Bakka A, Moberg I, Fetveit T, Mathisen O, Husby A, Sandvik O, Nesbakken A, Thiis-Evensen E, and Lothe RA. 2010. DNA sequence profiles of the colorectal cancer critical gene set KRAS-BRAF-PIK3CA-PTEN-TP53 related to age at disease onset. PLoS One 5:e13978. DOI 10.1371/journal.pone.0013978

Dekker JW, van den Broek CB, Bastiaannet E, van de Geest LG, Tollenaar RA, and Liefers GJ. 2011. Importance of the first postoperative year in the prognosis of elderly colorectal cancer patients. Ann Surg Oncol 18:1533-1539. DOI 10.1245/s10434-0111671-X

Fine JP, and Gray RJ. 1999. A Proportional Hazards Model for the Subdistribution of a Competing Risk. Publications of the American Statistical Association 94:496-509. DOI

Howlader N, Mariotto AB, Woloshin S, and Schwartz LM. 2014. Providing clinicians and patients with actual prognosis: cancer in the context of competing causes of death. $J$ Natl Cancer Inst Monogr 2014:255-264. DOI 10.1093/jncimonographs/lgu022

Kahn KL, Adams JL, Weeks JC, Chrischilles EA, Schrag D, Ayanian JZ, Kiefe CI, Ganz PA, Bhoopalam N, Potosky AL, Harrington DP, and Fletcher RH. 2010. Adjuvant chemotherapy use and adverse events among older patients with stage III colon cancer. Jama 303:1037-1045. DOI 10.1001/jama.2010.272

Kroenke CH, Neugebauer R, Meyerhardt J, Prado CM, Weltzien E, Kwan ML, Xiao J, and Caan BJ. 2016. Analysis of Body Mass Index and Mortality in Patients With Colorectal Cancer Using Causal Diagrams. JAMA Oncol 2:1137-1145. DOI 10.1001/jamaoncol.2016.0732

McCleary NJ, Meyerhardt JA, Green E, Yothers G, de Gramont A, Van Cutsem E, O'Connell M, Twelves CJ, Saltz LB, Haller DG, and Sargent DJ. 2013a. Impact of age on the efficacy of newer adjuvant therapies in patients with stage II/III colon cancer: findings from the ACCENT database. $J$ Clin Oncol 31:2600-2606. DOI $10.1200 /$ jco.2013.49.6638

McCleary NJ, Odejide O, Szymonifka J, Ryan D, Hezel A, and Meyerhardt JA. 2013b. Safety and effectiveness of oxaliplatin-based chemotherapy regimens in adults 75 years and older with colorectal cancer. Clin Colorectal Cancer 12:62-69. DOI 10.1016/j.clcc.2012.09.001

Merchant SJ, Nanji S, Brennan K, Karim S, Patel SV, Biagi JJ, and Booth CM. 2017. 
356

Management of stage III colon cancer in the elderly: Practice patterns and outcomes in the general population. Cancer 123:2840-2849. DOI

Noone AM, Lund JL, Mariotto A, Cronin K, McNeel T, Deapen D, and Warren JL. 2016. Comparison of SEER Treatment Data With Medicare Claims. Med Care 54:e55-64. DOI 10.1097/mlr.0000000000000073

Normand ST, Landrum MB, Guadagnoli E, Ayanian JZ, Ryan TJ, Cleary PD, and McNeil BJ. 2001. Validating recommendations for coronary angiography following acute myocardial infarction in the elderly: a matched analysis using propensity scores. $J$ Clin Epidemiol 54:387-398. DOI

Odden MC, Peralta CA, Haan MN, and Covinsky KE. 2012. Rethinking the association of high blood pressure with mortality in elderly adults: the impact of frailty. Arch Intern Med 172:1162-1168. DOI 10.1001/archinternmed.2012.2555

Papamichael D, Audisio R, Jc, Glimelius B, Sastre J, Mitry E, Van-Cutsem E, Gosney M, Kohne C, and Aapro M. 2009. Treatment of the elderly colorectal cancer patient: SIOG expert recommendations. Annals of Oncology 20:5-16. DOI

Robinson TN, Eiseman B, Wallace JI, Church SD, McFann KK, Pfister SM, Sharp TJ, and Moss M. 2009. Redefining geriatric preoperative assessment using frailty, disability and co-morbidity. Ann Surg 250:449-455. DOI 10.1097/SLA.0b013e3181b45598

Rosenberg AR, Kroon L, Chen L, Li CI, and Jones B. 2015. Insurance status and risk of cancer mortality among adolescents and young adults. Cancer 121:1279-1286. DOI $10.1002 /$ cncr.29187

Sanoff HK, Carpenter WR, Sturmer T, Goldberg RM, Martin CF, Fine JP, McCleary NJ, Meyerhardt JA, Niland J, Kahn KL, Schymura MJ, and Schrag D. 2012. Effect of adjuvant chemotherapy on survival of patients with stage III colon cancer diagnosed after age 75 years. J Clin Oncol 30:2624-2634. DOI 10.1200/jco.2011.41.1140

Sanoff HK, and Goldberg RM. 2007. Colorectal cancer treatment in older patients. Gastrointest Cancer Res 1:248-253. DOI

Schrag D, Cramer LD, Bach PB, and Begg CB. 2001. Age and adjuvant chemotherapy use after surgery for stage III colon cancer. J Natl Cancer Inst 93:850-857. DOI

Seymour M. 2004. Oxaliplatin, Fluorouracil, and Leucovorin as Adjuvant Treatment for Colon Cancer. New England Journal of Medicine 350:2343. DOI

Tournigand C, Andre T, Bonnetain F, Chibaudel B, Lledo G, Hickish T, Tabernero J, Boni C, Bachet JB, Teixeira $L$, and de Gramont A. 2012. Adjuvant therapy with fluorouracil and oxaliplatin in stage II and elderly patients (between ages 70 and 75 years) with colon cancer: subgroup analyses of the Multicenter International Study of Oxaliplatin, Fluorouracil, and Leucovorin in the Adjuvant Treatment of Colon Cancer trial. J Clin Oncol 30:3353-3360. DOI 10.1200/jco.2012.42.5645

Twelves C, Scheithauer W, McKendrick J, Seitz JF, Van Hazel G, Wong A, Diaz-Rubio E, Gilberg F, and Cassidy J. 2012. Capecitabine versus 5-fluorouracil/folinic acid as adjuvant therapy for stage III colon cancer: final results from the X-ACT trial with analysis by age and preliminary evidence of a pharmacodynamic marker of efficacy. Ann 
397

398

399

400

401

402

403

404

405

406

407

408

409

410

411

412

413

414

415

Oncol 23:1190-1197. DOI 10.1093/annonc/mdr366

Twelves C, Wong A, Nowacki MP, Abt M, Burris H, 3rd, Carrato A, Cassidy J, Cervantes A, Fagerberg J, Georgoulias V, Husseini F, Jodrell D, Koralewski P, Kroning H, Maroun J, Marschner N, McKendrick J, Pawlicki M, Rosso R, Schuller J, Seitz JF, Stabuc B, Tujakowski J, Van Hazel G, Zaluski J, and Scheithauer W. 2005. Capecitabine as adjuvant treatment for stage III colon cancer. N Engl J Med 352:26962704. DOI 10.1056/NEJMoa043116

van Erning FN, Bernards N, Creemers GJ, Vreugdenhil A, Lensen CJ, and Lemmens VE. 2014. Administration of adjuvant oxaliplatin to patients with stage III colon cancer is affected by age and hospital. Acta Oncol 53:975-980. DOI 10.3109/0284186x.2013.878470

van Erning FN, Creemers GJ, De Hingh IH, Loosveld OJ, Goey SH, and Lemmens VE. 2013. Reduced risk of distant recurrence after adjuvant chemotherapy in patients with stage III colon cancer aged 75 years or older. Ann Oncol 24:2839-2844. DOI 10.1093/annonc/mdt334

van Steenbergen LN, Steur M, Lemmens VE, Rutten HJ, van Spronsen DJ, and JanssenHeijnen ML. 2013. Minimal excess mortality for long-term colon cancer survivors in the Netherlands 1989-2008. Eur J Cancer 49:585-592. DOI 10.1016/j.ejca.2012.08.013 


\section{Figure 1 (on next page)}

70 was identified as a suitable cutoff age to define elderly CRC with 95\% $\mathrm{Cl} 68-72$.

Cox proportional hazard model with continued variable of age after transformation with restrict cubic spline method was plotted to examine the relationship between age and HR of colorectal cancer-specific death 76,858 eligible patients were included in the test cohort with dummy variables for each age to attain the HR of every age. Chow test was used to determine a suitable cutoff age. 


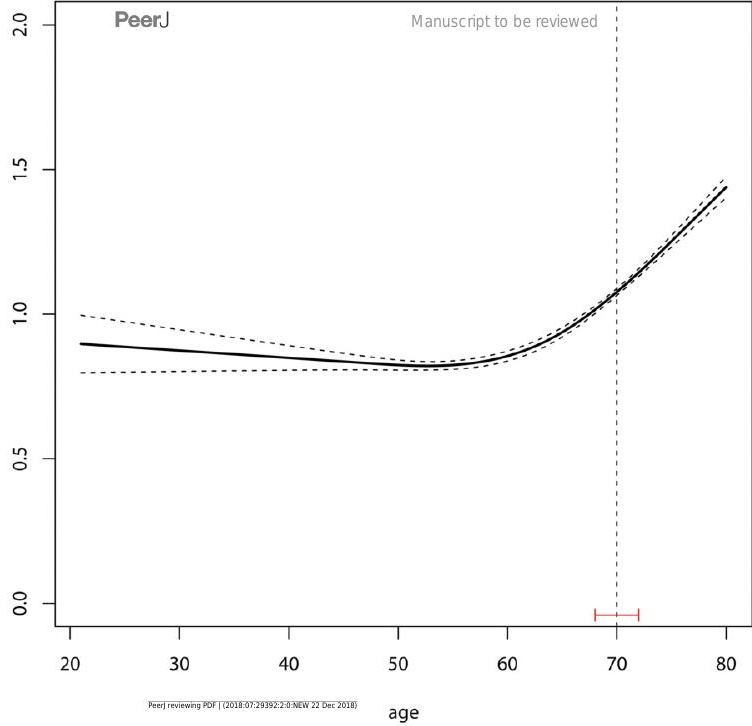




\section{Figure 2 (on next page)}

Gray method showed cumulative incidence curves of CSD and non-CSD in younger and older groups.

CSD: cancer-specific death. non-CSD: non-cancer-specific death. 


\section{Figure 3 (on next page)}

Forest plot of cancer-specific survival by patient subgroup in raw data.

HR: hazard ratio. 
Gender $\mathrm{Self}$

Female (35673)

Male (41185)

Marital status

Married (47437)

Single (11730)

Divorced (17691)

Race

White (60463)

Black (9057)

Others (7338)

Location

Left (46026)

Right (30832)

Histological type

Adenocarcinoma (68198)

Mucinous adenocarcinoma (7959)

Signet ring cell cancer (701)

Differentiated grade

Well (6208)

Moderate (57563)

Poor (13087)

$T$-Classification

T1 (6773)

T2 (13126)

T3 (47928)

T4 (9031)

$\mathrm{N}$-Classification

NO (45151)

N1 (20625)

N2 (11082)

nLN

0 (4076)

$0-2(1328)$

3-5 (3940)

6-11 (15302)

$\geqslant 12(52212)$

Stage TNM

I (16075)

|I (29076)

iil (31707)

Chemotherapy

No (41084)

Yes (35774)

Radiotherapy

No (63002)

Yes (13856)
Manuscript to be $\mathrm{HR}(95 \% \mathrm{CI})$

$1.53(1.45,1.60)$

$1.46(1.39,1.53)$

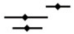

$1.55(1.48,1.62)$

$1.36(1.24,1.49)$

$1.37(1.28,1.46)$

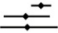

$1.52(1.46,1.58)$

$1.43(1.30,1.57)$

$1.44(1.28,1.62)$

$1.60(1.53,1.67)$

$1.36(1.29,1.44)$

$1.53(1.47,1.59)$

$1.23(1.12,1.36)$

$0.93(0.73,1.18)$

$1.57(1.36,1.81)$

$1.53(1.47,1.59)$

$1.28(1.20,1.36)$

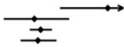

$1.91(1.63,2.23)$

$1.49(1.31,1.69)$

$1.52(1.46,1.59)$

$1.51(1.41,1.61)$

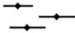

$1.57(1.49,1.66)$

$1.80(1.70,1.91)$

$1.63(1.53,1.73)$

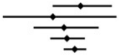

$1.52(1.35,1.70)$

$1.36(1.07,1.72)$

$1.42(1.25,1.62)$

$1.44(1.34,1.54)$

$1.48(1.42,1.55)$

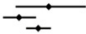

$1.72(1.53,1.94)$

$1.55(1.46,1.65)$

$1.66(1.59,1.74)$

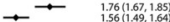

$1.56(1.49,1.64)$

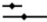

$1.51(1.46,1.57)$

$1.58(1.46,1.72)$ 
Figure 4 (on next page)

Kaplan-Meier estimate of cancer-specific survival. 


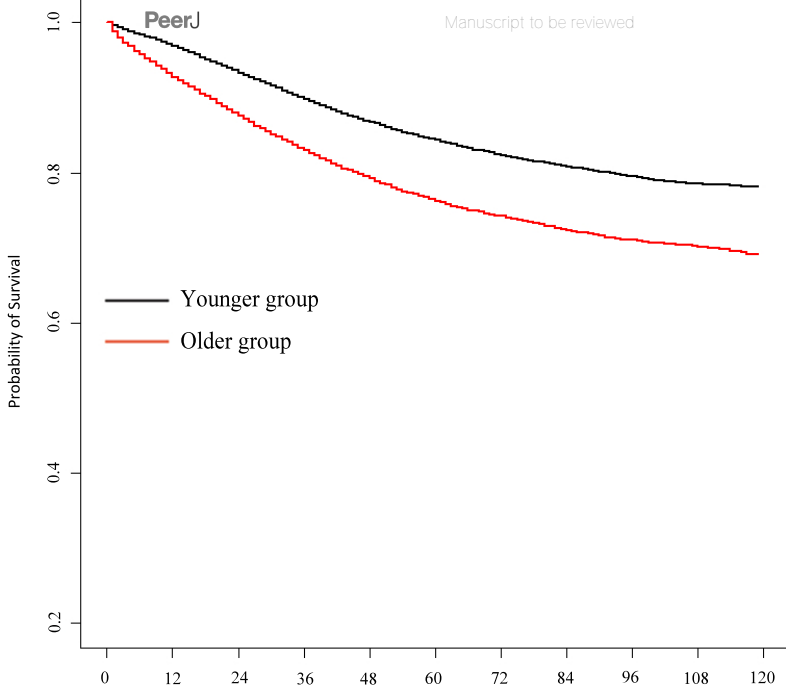




\section{Table $\mathbf{1}$ (on next page)}

The characteristics of 76,858 colorectal cancer patients in the younger and older groups. 


\begin{tabular}{|c|c|c|c|c|c|c|}
\hline \multirow[t]{3}{*}{ Characteristics } & Younger(<70yrs) & Older( $\geq 70 \mathrm{yrs})$ & \multirow{3}{*}{ SD } & Younger( $\leq 70 \mathrm{yrs})$ & Older $(\geq 70 y r s)$ & \multirow{3}{*}{ SD } \\
\hline & Before PSM & Before PSM & & After PSM & After PSM & \\
\hline & $N(\%)$ & $N(\%)$ & & $N(\%)$ & $N(\%)$ & \\
\hline \multicolumn{7}{|l|}{ Gender } \\
\hline Male & $22748(43.82)$ & $12925(51.82)$ & 0.57 & $11648(49.41)$ & 11924(50.59) & 0.02 \\
\hline Female & 29167(56.18) & $12018(48.18)$ & 0.92 & $12160(50.57)$ & $11884(49.43)$ & 0.02 \\
\hline \multicolumn{7}{|l|}{ Marital status } \\
\hline Married & $33210(63.97)$ & $14227(57.04)$ & 0.87 & $14646(50.78)$ & 14197(49.22) & 0.03 \\
\hline Single & $9416(18.14)$ & 2314(9.28) & 1.52 & $2370(50.60)$ & 2314(49.40) & 0.02 \\
\hline Divorced & 9289(17.89) & $8402(33.68)$ & 0.10 & $6792(48.21)$ & 7297(51.79) & 0.07 \\
\hline \multicolumn{7}{|l|}{ Race } \\
\hline White & 39981(77.01) & 20482(82.12) & 0.68 & 19317(49.92) & 19379(50.08) & $<0.01$ \\
\hline Black & $6768(13.04)$ & $2289(9.18)$ & 1.14 & 2339(50.55) & $2288(49.45)$ & 0.02 \\
\hline Others & $5166(9.95)$ & $2172(8.71)$ & 0.89 & 2152(50.13) & 2141(49.87) & 0.01 \\
\hline \multicolumn{7}{|l|}{ Location } \\
\hline Left & $33719(64.95)$ & $12307(49.34)$ & 1.05 & 12470(50.69) & 12131(49.31) & 0.03 \\
\hline Right & $18196(35.05)$ & $12636(50.66)$ & 0.37 & $11338(49.26)$ & $11677(50.74)$ & 0.03 \\
\hline \multicolumn{7}{|l|}{ Histological type } \\
\hline Adenocarcinoma & $46246(89.08)$ & 21952(88.01) & 0.76 & $21056(50.23)$ & 20862(49.77) & 0.01 \\
\hline $\begin{array}{l}\text { Mucinous } \\
\text { adenocarcinoma }\end{array}$ & 5181(9.98) & $2778(11.14)$ & 0.63 & $2538(48.14)$ & 2734(51.86) & 0.07 \\
\hline $\begin{array}{l}\text { Signet ring cell } \\
\text { cancer }\end{array}$ & $488(0.94)$ & $213(0.85)$ & 0.85 & $214(50.23)$ & 212(49.77) & 0.01 \\
\hline \multicolumn{7}{|l|}{ Differentiated grade } \\
\hline Well & $4225(8.14)$ & 1983(7.95) & 0.77 & 1952(50.03) & 1950(49.97) & $<0.01$ \\
\hline Moderate & $39154(75.42)$ & $18409(73.80)$ & 0.77 & $17677(50.41)$ & 17391(49.59) & 0.02 \\
\hline Poor & $8536(16.44)$ & $4551(18.25)$ & 0.64 & $4179(48.33)$ & $4467(51.67)$ & 0.07 \\
\hline \multicolumn{7}{|l|}{ T-classification ${ }^{a}$} \\
\hline $\mathrm{T} 1$ & $4594(8.85)$ & $2179(8.74)$ & 0.76 & $2178(50.09)$ & 2170(49.91) & $<0.01$ \\
\hline $\mathrm{T} 2$ & $8546(16.46)$ & $4580(18.36)$ & 0.63 & 4429(49.92) & 4443(50.08) & $<0.01$ \\
\hline $\mathrm{T} 3$ & $32608(62.81)$ & $15320(61.42)$ & 0.77 & $14518(50.25)$ & $14376(49.75)$ & 0.01 \\
\hline $\mathrm{T} 4$ & $6167(11.88)$ & $2864(11.48)$ & 0.79 & $2683(48.76)$ & $2819(51.24)$ & 0.05 \\
\hline \multicolumn{7}{|l|}{$\mathrm{N}$-classification ${ }^{\text {a }}$} \\
\hline No & $29285(56.41)$ & $15866(63.61)$ & 0.62 & $15072(50.35)$ & 14863(49.65) & 0.01 \\
\hline N1 & $14542(28.01)$ & $6083(24.39)$ & 0.90 & $5764(49.11)$ & $5972(50.89)$ & 0.04 \\
\hline $\mathrm{N} 2$ & $8088(15.58)$ & 2994(12.00) & 1.04 & 2972(49.99) & $2973(50.01)$ & $<0.01$ \\
\hline \multicolumn{7}{|l|}{$n L N$} \\
\hline 0 & $2733(5.26)$ & $1343(5.38)$ & 0.73 & $1324(50.06)$ & 1321(49.94) & $<0.01$ \\
\hline $1-2$ & $872(1.68)$ & $456(1.83)$ & 0.66 & $407(47.22)$ & $455(52.78)$ & 0.11 \\
\hline $3-5$ & $2518(4.85)$ & $1422(5.70)$ & 0.58 & $1314(50.00)$ & $1314(50.00)$ & $<0.01$ \\
\hline $6-11$ & $9808(18.89)$ & $5494(22.03)$ & 0.59 & 5013(49.18) & $5180(50.82)$ & 0.03 \\
\hline
\end{tabular}




\begin{tabular}{|c|c|c|c|c|c|c|}
\hline$\geq 12$ & $35984(69.31)$ & $16228(65.06)$ & 0.82 & $15750(50.34)$ & $15538(49.66)$ & 0.01 \\
\hline \multicolumn{7}{|c|}{ Stage ${ }^{a}$} \\
\hline I & $10334(19.91)$ & $5741(23.02)$ & 0.60 & $5647(50.23)$ & $5596(49.77)$ & 0.01 \\
\hline II & $18951(36.50)$ & $10125(40.59)$ & 0.64 & $9425(50.42)$ & $9267(49.58)$ & 0.02 \\
\hline III & $22630(43.59)$ & $9077(36.39)$ & 0.95 & $8736(49.41)$ & $8945(50.59)$ & 0.02 \\
\hline \multicolumn{7}{|l|}{$\mathrm{CT}$} \\
\hline No & $24094(46.41)$ & $16990(68.12)$ & 0.35 & $15580(49.56)$ & $15856(50.44)$ & 0.02 \\
\hline Yes & $27821(53.59)$ & $7953(31.88)$ & 1.34 & $8228(50.85)$ & 7952(49.15) & 0.03 \\
\hline \multicolumn{7}{|l|}{ RT } \\
\hline No & $40769(78.53)$ & $22233(89.14)$ & 0.62 & 20949(49.82) & 21098(50.18) & 0.01 \\
\hline Yes & $11146(21.47)$ & $2710(10.86)$ & 1.53 & 2859(51.34) & 2710(48.66) & 0.05 \\
\hline
\end{tabular}

$1 \quad$ a Stage TNM, T, N-classification to $7^{\text {th }}$ edition of AJCC staging system.

2 All statistical tests were two-sided.

3 Abbreviations: PSM: propensity score matching; SD: standardized difference; nLN: number of lymph nodes;

4 CT: chemotherapy treatment; RT: radiotherapy treatment.

5 Left includes rectum, rectosigmoid junction, sigmoid colon, descending colon and splenic flexure.

6 Right includes transverse colon, hepatic flexure, ascending colon, cecum, and appendix. 


\section{Table 2 (on next page)}

Univariate and Multivariate Analysis of Colorectal Cancer-Specific Death of 47,616 patients after PSM. 


\begin{tabular}{|c|c|c|c|c|}
\hline \multirow[t]{2}{*}{ Risk Factors } & \multicolumn{2}{|c|}{ Univariate analysis } & \multicolumn{2}{|c|}{ Multivariate analysis } \\
\hline & SHR $(95 \% C I)$ & $P^{\mathrm{b}}$ & SHR $(95 \% C I)$ & $P^{\mathrm{b}}$ \\
\hline \multicolumn{5}{|l|}{ Age } \\
\hline$<70$ & 1 & & 1 & \\
\hline$\geq 70$ & $1.47(1.41-1.53)$ & $<0.001$ & $1.50(1.44-1.56)$ & $<0.001$ \\
\hline \multicolumn{5}{|l|}{ Gender } \\
\hline Female & 1 & & 1 & \\
\hline Male & $1.06(1.02-1.10)$ & 0.005 & $1.11(1.06-1.16)$ & $<0.001$ \\
\hline \multicolumn{5}{|l|}{ Marital status } \\
\hline Married & 1 & & 1 & \\
\hline Unmarried & $1.48(1.39-1.58)$ & $<0.001$ & $1.27(1.19-1.36)$ & $<0.001$ \\
\hline Divorced & $1.35(1.30-1.42)$ & $<0.001$ & $1.25(1.19-1.31)$ & $<0.001$ \\
\hline \multicolumn{5}{|l|}{ Race } \\
\hline White & 1 & & 1 & \\
\hline Black & $1.31(1.23-1.40)$ & $<0.001$ & $1.26(1.18-1.35)$ & $<0.001$ \\
\hline Other & $0.95(0.88-1.02)$ & 0.176 & $0.90(0.84-0.97)$ & 0.008 \\
\hline \multicolumn{5}{|l|}{ Location } \\
\hline Left & 1 & & 1 & \\
\hline Right & $0.93(0.89-0.97)$ & $<0.001$ & $1.01(0.96-1.06)$ & 0.759 \\
\hline \multicolumn{5}{|l|}{ Histology } \\
\hline Adenocarcinoma & 1 & & 1 & \\
\hline $\begin{array}{l}\text { Mucinous adenocarci } \\
\text { noma }\end{array}$ & $1.14(1.07-1.21)$ & $<0.001$ & $1.08(1.01-1.15)$ & 0.018 \\
\hline $\begin{array}{l}\text { Signet } \\
\text { ring cell carcinoma }\end{array}$ & $3.05(2.62-3.54)$ & $<0.001$ & $1.48(1.26-1.74)$ & $<0.001$ \\
\hline \multicolumn{5}{|l|}{ Differentiated grade } \\
\hline Grade I & 1 & & 1 & \\
\hline Grade II & $1.43(1.31-1.56)$ & $<0.001$ & $1.27(1.16-1.39)$ & $<0.001$ \\
\hline Grade III & $2.45(2.23-2.69)$ & $<0.001$ & $1.59(1.45-1.76)$ & $<0.001$ \\
\hline \multicolumn{5}{|l|}{ T-classification ${ }^{a}$} \\
\hline $\mathrm{T} 1$ & 1 & & 1 & \\
\hline $\mathrm{T} 2$ & $0.73(0.65-0.83)$ & $<0.001$ & $0.96(0.85-1.08)$ & 0.472 \\
\hline $\mathrm{T} 3$ & $2.15(1.95-2.37)$ & $<0.001$ & $2.19(1.98-2.43)$ & $<0.001$ \\
\hline $\mathrm{T} 4$ & $5.46(4.92-6.05)$ & $<0.001$ & $4.77(4.27-5.33)$ & $<0.001$ \\
\hline \multicolumn{5}{|l|}{$\mathrm{N}$ - classification ${ }^{a}$} \\
\hline N0 & 1 & & 1 & \\
\hline N1 & $2.33(2.22-2.44)$ & $<0.001$ & $2.16(2.05-2.28)$ & $<0.001$ \\
\hline N2 & $4.48(4.26-4.71)$ & $<0.001$ & $4.05(3.81-4.30)$ & $<0.001$ \\
\hline \multicolumn{5}{|l|}{$\mathrm{nLN}$} \\
\hline 0 & 1 & & 1 & \\
\hline $0-2$ & $0.49(0.42-0.58)$ & $<0.001$ & $0.39(0.33-0.46)$ & $<0.001$ \\
\hline
\end{tabular}




\begin{tabular}{lllll}
\hline $3-5$ & $0.61(0.55-0.68)$ & $<0.001$ & $0.43(0.39-0.48)$ & $<0.001$ \\
6-11 & $0.56(0.52-0.61)$ & $<0.001$ & $0.33(0.30-0.36)$ & $<0.001$ \\
$\geq 12$ & $0.47(0.44-0.51)$ & $<0.001$ & $0.24(0.22-0.26)$ & $<0.001$ \\
CT & & & & \\
No & 1 & & 1 & \\
Yes & $1.70(1.64-1.77)$ & $<0.001$ & $0.84(0.80-0.89)$ & $<0.001$ \\
RT & 1 & & & \\
No & $1.35(1.28-1.43)$ & $<0.001$ & $1.15(1.08-1.23)$ & $<0.001$ \\
Yes & & & & \\
\hline
\end{tabular}

$1{ }^{\mathrm{a}} \mathrm{T}$ classification according to $7^{\text {th }}$ AJCC staging system.

$2 \quad \mathrm{~b} P$ values obtained from the $\chi 2$ test. All statistical tests were two-sided.

3 Abbreviations: PSM: propensity score matching; CSD: cancer specific death; nLN: number of lymph nodes;

4 CT: chemotherapy treatment; RT: radiotherapy treatment; SHR: subdistribution hazard ratio.

5 Left includes rectum, rectosigmoid junction, sigmoid colon, descending colon and splenic flexure.

6 Right includes transverse colon, hepatic flexure, ascending colon, cecum, and appendix. 


\section{Table 3(on next page)}

Univariate and Multivariate Analysis of Colorectal Cancer-Specific Survival of 47,616 patients after PSM. 


\begin{tabular}{|c|c|c|c|c|}
\hline \multirow[t]{2}{*}{ Risk Factors } & \multicolumn{2}{|c|}{ Univariate analysis } & \multicolumn{2}{|c|}{ Multivariate analysis } \\
\hline & HR $(95 \% \mathrm{CI})$ & $\mathrm{Pb}$ & HR $(95 \% \mathrm{CI})$ & $\mathrm{Pb}$ \\
\hline \multicolumn{5}{|l|}{ Age } \\
\hline$<70$ & 1 & & 1 & \\
\hline$\geq 70$ & $1.57(1.51-1.64)$ & $<0.001$ & $1.64(1.57-1.70)$ & $<0.001$ \\
\hline \multicolumn{5}{|l|}{ Gender } \\
\hline Female & 1 & & 1 & \\
\hline Male & $1.08(1.04-1.13)$ & $<0.001$ & $1.14(1.10-1.19)$ & $<0.001$ \\
\hline \multicolumn{5}{|l|}{ Marital status } \\
\hline Married & 1 & & 1 & \\
\hline Unmarried & $1.54(1.44-1.64)$ & $<0.001$ & $1.33(1.25-1.42)$ & $<0.001$ \\
\hline Divorced & $1.39(1.33-1.46)$ & $<0.001$ & $1.29(1.23-1.35)$ & $<0.001$ \\
\hline \multicolumn{5}{|l|}{ Race } \\
\hline White & 1 & & 1 & \\
\hline Black & $1.34(1.26-1.43)$ & $<0.001$ & $1.29(1.21-1.38)$ & $<0.001$ \\
\hline Other & $0.93(0.86-1.00)$ & 0.06 & $0.87(0.81-0.94)$ & $<0.001$ \\
\hline \multicolumn{5}{|l|}{ Location } \\
\hline Left & 1 & & 1 & \\
\hline Right & $0.93(0.89-0.97)$ & 0.001 & $1.01(0.96-1.06)$ & 0.697 \\
\hline \multicolumn{5}{|l|}{ Histology } \\
\hline Adenocarcinoma & 1 & & 1 & \\
\hline $\begin{array}{l}\text { Mucinous adenocarci } \\
\text { noma }\end{array}$ & $1.13(1.07-1.21)$ & $<0.001$ & $1.07(1.01-1.14)$ & 0.033 \\
\hline $\begin{array}{l}\text { Signet } \\
\text { ring cell carcinoma }\end{array}$ & $3.10(2.69-3.58)$ & $<0.001$ & $1.55(1.34-1.79)$ & $<0.001$ \\
\hline \multicolumn{5}{|l|}{ Differentiated grade } \\
\hline Grade I & 1 & & 1 & \\
\hline Grade II & $1.43(1.31-1.57)$ & $<0.001$ & $1.30(1.19-1.42)$ & $<0.001$ \\
\hline Grade III & $2.47(2.25-2.72)$ & $<0.001$ & $1.64(1.49-1.81)$ & $<0.001$ \\
\hline \multicolumn{5}{|l|}{ T-classification ${ }^{a}$} \\
\hline $\mathrm{T} 1$ & 1 & & 1 & \\
\hline $\mathrm{T} 2$ & $0.73(0.64-0.82)$ & $<0.001$ & $0.96(0.85-1.08)$ & 0.488 \\
\hline $\mathrm{T} 3$ & $2.15(1.95-2.37)$ & $<0.001$ & $2.24(2.02-2.49)$ & $<0.001$ \\
\hline $\mathrm{T} 4$ & $5.57(5.02-6.17)$ & $<0.001$ & $5.01(4.49-5.59)$ & $<0.001$ \\
\hline \multicolumn{5}{|l|}{$\mathrm{N}$ - classification ${ }^{a}$} \\
\hline N0 & 1 & & 1 & \\
\hline N1 & $2.33(2.23-2.45)$ & $<0.001$ & $2.22(2.10-2.34)$ & $<0.001$ \\
\hline $\mathrm{N} 2$ & $4.53(4.31-4.77)$ & $<0.001$ & $4.24(4.00-4.49)$ & $<0.001$ \\
\hline \multicolumn{5}{|l|}{$\mathrm{nLN}$} \\
\hline 0 & 1 & & 1 & \\
\hline $0-2$ & $0.48(0.40-0.56)$ & $<0.001$ & $0.37(0.31-0.43)$ & $<0.001$ \\
\hline
\end{tabular}




\begin{tabular}{|c|c|c|c|c|}
\hline $3-5$ & $0.58(0.53-0.65)$ & $<0.001$ & $0.41(0.37-0.46)$ & $<0.001$ \\
\hline $6-11$ & $0.54(0.49-0.58)$ & $<0.001$ & $0.30(0.28-0.33)$ & $<0.001$ \\
\hline$\geq 12$ & $0.44(0.41-0.48)$ & $<0.001$ & $0.22(0.20-0.23)$ & $<0.001$ \\
\hline \multicolumn{5}{|l|}{$\mathrm{CT}$} \\
\hline No & 1 & & 1 & \\
\hline Yes & $1.65(1.59-1.72)$ & $<0.001$ & $0.78(0.75-0.82)$ & $<0.001$ \\
\hline \multicolumn{5}{|l|}{ RT } \\
\hline No & 1 & & 1 & \\
\hline Yes & $1.32(1.25-1.40)$ & $<0.001$ & $1.14(1.07-1.22)$ & $<0.001$ \\
\hline \multicolumn{5}{|c|}{${ }^{\text {a }} \mathrm{T}$ classification according to $7^{\text {th }}$ AJCC staging system. } \\
\hline \multicolumn{5}{|c|}{${ }^{\mathrm{b}} P$ values obtained from the $\chi 2$ test. All statistical tests were two-sided. } \\
\hline \multicolumn{5}{|c|}{ Abbreviations: PSM: propensity score matching; CSD: cancer specific death; nLN: number of lymph nodes; } \\
\hline \multicolumn{5}{|c|}{ CT: chemotherapy treatment; RT: radiotherapy treatment; HR: hazard ratio. } \\
\hline \multicolumn{5}{|c|}{ Left includes rectum, rectosigmoid junction, sigmoid colon, descending colon and splenic flexure. } \\
\hline \multicolumn{5}{|c|}{ Right includes transverse colon, hepatic flexure, ascending colon, cecum, and appendix. } \\
\hline
\end{tabular}

